\title{
TEMPORALIDAD Y RELACIONES DE INCLUSIÓN: EL PROCESAMIENTO DE LA REGLA CONDICIONAL EN LA TAREA DE SELECCIÓN Y SUS DIFICULTADES
}

Temporality and inclusion relations: conditional rule processing in selection task and its difficulties

\author{
Miguel López Astorga*
}

Resumen

Algunas versiones de la tarea de selección de las cuatro tarjetas de Peter Wason son realizadas correctamente por los sujetos experimentales, pero en otras no sucede lo mismo. En este trabajo, intentamos explicar este problema y argumentamos que los sujetos seleccionan las tarjetas válidas lógicamente cuando las versiones cuentan con (i) una regla temporal o (ii) una regla con un término que puede ser entendido como subconjunto del otro término. De esta manera, defendemos que los problemas de la tarea de Wason no son problemas relacionados con la inferencia lógica, sino problemas referentes al proceso de construcción de representaciones mentales.

Palabras clave: Inferencia, representación mental, subconjunto, temporalidad.

Abstract

Some versions of Peter Wason's four-cards selection task are executed correctly by experimental subjects, but other versions are not. In this paper, I try to explain this problem and I argue that subjects select logically valid cards when the versions have (i) a temporal rule or (ii) a rule with a term that can be understood as subset of the other term. Thus, I hold problems of Wason's task are not logical inference problems, but problems about construction of mental representations process.

Key words: Inference, mental representations, subset, temporality.

INTRODUCCIÓN: EL PROBLEMA

El problema que vamos a abordar en estas páginas no es otro sino el de la -a pesar de ser bien conocida - muy controvertida y discutida tarea de selección de las cuatro tarjetas de Peter Wason (WST, Wason Selection Task: "Tarea de selección de Wason"). Evidentemente, podemos encontrar exhaustivas y claras descripciones de WST en los primeros textos del propio Wason al respecto (1966-1968). Empero, hemos ofrecido en otro trabajo (2008) una explicación en español de sus características y de las dificultades que encierra. Recurrimos a tal explicación de nuevo para exponer de un modo más nítido la problemática que nos va a ocupar en lo que sigue.

La tarea WST se basa en cuatro tarjetas que tienen una característica en común: por una de sus caras presentan una letra y por la otra un número. Los participantes en ella, sin embargo, sólo pueden observar una de las caras de cada una 
de esas cuatro tarjetas. Podemos suponer, por ejemplo, que están colocadas en una mesa y que sólo es posible ver, por tanto, los anversos de las tarjetas que se muestran y no sus reversos. En nuestra descripción de WST (2008), las tarjetas mostraban estas letras y estos números: " $A$ " era la letra que aparecía en la primera, "C" la letra que correspondía a la segunda, " 2 " el número que se podía apreciar en la tercera, y " 3 " el número que figuraba en la cuarta. Ante esta situación, lo que el participante tenía que hacer era indicar qué tarjeta o qué tarjetas había que girar para comprobar si la regla que se indica a continuación era verdadera o falsa

Si en una tarjeta hay una vocal en una cara, entonces hay un número par en la otra (2008:79).

Sin duda, esta tarea parece ser bastante sencilla y, precisamente por ello, cabe preguntarse por qué es realmente un problema. La respuesta no es compleja. Wason (1966) deseaba comprobar si la mente humana es racional y si las personas, cuando razonamos, nos atenemos a las prescripciones de la lógica formal. No obstante, los resultados que obtuvieron sus participantes en WST parecieron demostrar que ni la mente humana es racional ni las personas realizamos inferencias acordes con la lógica clásica, ya que muy pocos sujetos la resolvieron adecuadamente. De hecho, WST llegó a convertirse en una de las tareas más controvertidas en el ámbito de la ciencia cognitiva y de la psicología del razonamiento pues, incluso hoy, más de cuarenta años después de que Wason la planteara por primera vez, se continúa investigando sobre ella, se escribe acerca de sus consecuencias y sus dificultades y se discute, desde diferentes marcos teóricos, cuáles pueden ser las causas de sus resultados no esperados . ${ }^{1}$ Hemos de tener en cuenta, además, que WST ha sido replicada en incontables ocasiones ${ }^{2}$ y que casi siempre ha arrojado resultados similares, puesto que la mayoría de los sujetos que se enfrentan a ella no parecen ser capaces de señalar las tarjetas correctas desde el punto de vista lógico.

No es difícil explicar cuáles son las tarjetas lógicamente válidas en WST. En cualquiera de los trabajos citados hasta el momento podemos hallar una explicación al respecto. Sin embargo, podemos revisar aquí también, con fines básicamente ilustrativos, cada una de las tarjetas por separado para intentar determinar si debe ser girada o no. Procedemos, pues, a tal revisión

"A": evidentemente, tenemos que observar lo que aparece en su lado oculto, ya que puede tener tanto "2" como "3" y la regla no sería verdadera en este último caso.

\footnotetext{
${ }^{1}$ Ejemplos de la relevancia que en la actualidad sigue teniendo WST pueden ser los trabajos de Evans (2008) donde se reflexiona sobre ella para intentar averiguar cuáles son los verdaderos procesos que acontecen en la mente humana cuando se razona, los de Rutherford (2009) que utiliza versiones de WST con el propósito de verificar las funciones intelectuales que pueden realizar sin demasiadas dificultades los sujetos diagnosticados como autistas, o Beller (2010), quien se hace referencia a la WST con el objetivo de determinar si, además de la lógica clásica, existe una lógica deóntica relativa al razonamiento con permisos y prohibiciones.

${ }^{2}$ Para profundizar en este asunto, véase, por ejemplo, Santamaría (1995) o López Astorga (2004-2008).
} 
"C": es una tarjeta irrelevante, pues, tenga lo que tenga detrás, la regla continuará siendo verdadera. Recordemos que la regla establece que las vocales deben tener en su lado oculto números pares, no que las consonantes tengan que presentar números impares.

" 2 ": ésta es la tarjeta más controvertida, y ello debido a que suele ser elegida por los participantes sin tener que hacerlo. En realidad, y a pesar de que aparentemente parece lo contrario, esta tarjeta tampoco es informativa. La regla establece que las vocales deben ir acompañadas, en su otra cara, por números pares, no que los números pares deban tener en su otro lado una vocal. El condicional se tiene que leer en una sola dirección y no es, por tanto, un bicondicional. De este modo, es fácil notar que esta tarjeta no es apropiada, puesto que, aunque tuviera una consonante en la cara que no podemos ver, la regla seguiría siendo verdadera.

"3": esta tarjeta, sin embargo, sí es informativa, ya que, para que la regla siga siendo válida, no puede tener una vocal en su cara inversa.

Tenemos, así, que la respuesta correcta es, para este ejemplo concreto, la combinación "A" y "3". No obstante, la respuesta mayoritaria de los participantes, como se puede comprobar en la literatura de la ciencia cognitiva, es la combinación "A" y "2", siendo la tarjeta "A" exclusivamente la segunda respuesta más elegida. Y lo más llamativo de este fenómeno es que los participantes se equivocan, incluso, cuando se trata de sujetos con formación académica universitaria relacionada con el ámbito lógico o matemático, porque, habitualmente —como hemos indicado- son muy pocos los participantes que prefieren las tarjetas válidas lógicamente.

Empero, el propósito de este trabajo no es presentar una explicación de por qué ocurre esto y la mayor parte de los sujetos que se enfrentan a WST yerran en sus elecciones. En otros trabajos nuestros (2004-2008-2008b) ya hemos argumentado al respecto y hemos expuesto nuestras tesis para lo que a este asunto se refiere. En nuestra opinión, la tarea es excesivamente abstracta y el contexto que se describe en ella es tan pobre que impide que el sujeto pueda entenderla apropiadamente y pueda notar que la regla es un enunciado condicional. Ello es, sin más, lo que sucede con WST, pudiendo los participantes interpretar —en ocasiones - que los dos términos del enunciado, la vocal y el número par, no están vinculados por una relación condicional, sino, por ejemplo, por una conjunción, lo cual sería compatible con la selección mayoritaria, las tarjetas " $\mathrm{A}$ " $\mathrm{y}$ " 2 " de nuestro ejemplo (2008b).

Lo que nos interesa, ahora, es otro fenómeno asociado a WST y que también puede despertar, en gran medida, la curiosidad intelectual. Como nos cuenta Santamaría (1995), durante la década de los setenta se defendió algo con lo que nuestras hipótesis son totalmente consistentes: si la tarea no se le presenta a los sujetos del modo abstracto como Peter Wason se la propuso originalmente a sus participantes, sino con un contenido temático concreto, los resultados mejoran significativamente. Nuestros planteamientos anteriores son, como decimos, coherentes con esta idea porque, si los problemas de WST están relacionados con su pobre contexto y su grado de abstracción, - tal y como defendimos - un enriquecimiento del contexto y un contenido concreto 
deben, necesariamente, aumentar el número de respuestas correctas desde el punto de vista lógico. Según Santamaría, entre los investigadores pareció difundirse una tesis que asumieron de modo más o menos implícito y según la cual, los sujetos por lo general, realizan cotidianamente inferencias a partir de contenidos concretos, no a partir de materiales abstractos. Muchos estudios en esta línea, nos dice, parecieron apoyar tal tesis porque algunas versiones con contenido temático concreto obtuvieron, inicialmente, muy buenos resultados. ${ }^{3}$

Santamaría también nos recuerda que la satisfacción por los resultados logrados no se prolongó por mucho tiempo y que en ello desempeñó un papel decisivo el trabajo de Manktelow y Evans (1979) quien comprobó que el contenido temático no necesariamente mejora la ejecución de WST. Y es que, a partir de él, se puede decir como se deriva de la interpretación de Santamaría - que, aunque, a veces, el contenido temático concreto incrementa las selecciones correctas desde el punto de vista lógico en WST, no todo contenido temático logra tal efecto, sino, exclusivamente, el contenido temático con determinadas características.

Por tanto, un problema abierto en el presente con respecto a WST es el relativo a qué características debe poseer un contenido temático para mejorar su ejecución. En este trabajo, vamos a intentar resolverlo.

TEMPORALIDAD Y RELACIONES DE INCLUSIÓN COMO ELEMENTOS FUNDAMENTALES DEL CONTENIDO QUE MEJORAN LOS RESULTADOS DE WST

Diversos enfoques teóricos han tratado de resolver y de explicar por qué algunos tipos de contenido aumentan las respuestas correctas en WST y otros no. Analizando versiones con contenido temático ejecutadas correctamente, se ha postulado la existencia de mecanismos mentales no estrictamente lógico-formales que ayudan a elegir las tarjetas adecuadas y que permiten extraer conclusiones sin atenerse a las normas o prescripciones de la lógica formal clásica. Según estos enfoques, tales mecanismos han permanecido desconocidos para los investigadores hasta tiempos recientes y, por consecuencia, no han sido tenidos en cuenta en tanto elementos o factores que pueden incidir en tareas de razonamiento como WST.

Ejemplos significativos de estos enfoques pueden ser la teoría de los esquemas pragmáticos de razonamiento (Cheng y Holyoak, 1985; Cheng, Holyoak, Nisbett y Oliver, 1986), teoría que defiende que los seres humanos contamos con esquemas de razonamiento pragmático en nuestra mente y que dichos esquemas son activados cuando el contexto de la versión de WST, en particular, describe una situación de índole práctica relacionada con permisos o con obligaciones. $\mathrm{O}$, la teoría de los contratos sociales (Cosmides, 1989; Fiddick, Cosmides y Tooby, 2000) según la cual, debido a que los seres humanos poseemos reglas mentales

${ }^{3}$ Siguiendo el argumento de Santamaría, podemos decir que trabajos representativos en este sentido bien pueden ser los de Wason y Shapiro (1971), Johnson-Laird, Legrenzi y Legrenzi (1972) o Van Duyne (1974). 
adaptativas que regulan las situaciones de intercambio, sólo se ejecutan correctamente aquellas versiones de WST en las que se obtiene un beneficio a cambio de cumplir con un requisito.

No obstante, este tipo de enfoque no nos parece aceptable, y ello ya lo hemos puesto de manifiesto en diversos trabajos (2004-2010). Desde nuestra óptica, los resultados de los experimentos que se presentan para apoyar los supuestos esenciales de estas teorías no suelen ser concluyentes ya que, generalmente, son versiones de WST que van acompañadas de instrucciones que, al estar expresadas con mayor claridad que en otras versiones, permiten una mejor comprensión de la labor a realizar y, por tanto, conducen a resultados más óptimos. De esta manera, si existen mecanismos mentales como los propuestos por los marcos teóricos en esta línea, ello no está en absoluto demostrado ni por los argumentos teóricos en que se apoyan ni por los resultados experimentales que suelen proponer como evidencias.

Somos conscientes, sin embargo, de que estas críticas destructivas deben ir acompañadas de alternativas explicativas que, de algún modo, proporcionen indicios de los posibles motivos por los que, a pesar de que con otras versiones no sucede lo mismo, algunas versiones de WST con contenido temático son resueltas adecuadamente por los participantes. Por lo que a esto se refiere, estamos convencidos de que, en la literatura sobre WST, podemos hallar diversas investigaciones que pueden ayudarnos en nuestra búsqueda de una explicación. ${ }^{4}$ Son de suma trascendencia, para nosotros, por ejemplo, investigaciones como las de Margolis (1987-2000) o Nickerson (1996), las cuales plantean que la ambigüedad contextual y la poca descripción del escenario en algunas versiones de WST son los auténticos factores responsables de sus resultados negativos. Del mismo modo, también son de enorme interés, según entendemos, los argumentos de Yachanin y Tweney (1982) referentes a que la respuesta válida desde un punto de vista lógico se ve favorecida en WST cuando en la versión correspondiente se dan dos elementos importantes

1.- La regla no se presenta como una regla hipotética cuya verdad o falsedad es necesario comprobar, sino como una regla verdadera y aceptada, con respecto a la que hay que detectar posibles infractores.

2.- El participante tiene que asumir el rol de una personalidad con autoridad que tiene por misión detectar si otros individuos infringen la regla propuesta.

Las investigaciones mencionadas son, obviamente, relevantes y, por supuesto, admitimos que los elementos y factores a los que hacen referencia deben tener su importancia relativa en los procesos inferenciales que conducen a los participantes a elegir unas tarjetas y no otras. De hecho, como hemos apuntado más arriba, hemos defendido en trabajos previos que la pobreza contextual y la ambiguiedad son aspectos claves para comprender por qué las versiones abstractas iniciales de WST no son resueltas adecuadamente. Consideramos, empero, que existen otros dos factores que no

\footnotetext{
${ }^{4}$ A muchas de estas investigaciones ya hicimos mención en López Astorga (2004).
} 
he mencionados hasta el momento y que pueden ser aún más decisivos en cuanto instancias explicativas de las razones por las que sólo ciertos contenidos temáticos incrementan las respuestas correctas en WST. Tales aspectos, que caracterizan y se encuentran presentes únicamente en las versiones que se ejecutan correctamente, son la temporalidad de la regla y la relación de inclusión entre el antecedente y el consecuente del condicional. Desde nuestro punto de vista, estos aspectos son esenciales en esta problemática, ya que, en prácticamente todas las versiones de WST en las que se obtienen buenos resultados, podemos observar que: a) Hay una distancia temporal entre el antecedente y el consecuente, o b) Uno de esos dos términos puede ser considerado un subconjunto del otro.

En lo que resta, vamos a revisar las versiones más emblemáticas de WST que podemos encontrar para demostrar que nuestra tesis es cierta, esto es, que todas las versiones de WST en las que los participantes se inclinan por las tarjetas adecuadas presentan una regla con una dimensión temporal o con una relación de inclusión. Sin embargo, antes de comenzar, vamos a describir un trabajo en el que ya se consideró la posibilidad de que la temporalidad influyera en los resultados de WST, pues pensamos que los resultados de tal trabajo son consistentes con nuestro planteamiento. Posteriormente, pasaremos a la revisión directa de distintas versiones de WST propuestas en los últimos tiempos, con el fin de demostrar nuestras afirmaciones.

\section{ALKHALIFA Y SU VISIÓN DEL TIEMPO}

Alkhalifa (2001) plantea que pueden ser dos las variables que influyen en la selección de tarjetas en WST. La primera es similar al segundo elemento que Yachanin y Tweney (1982) consideran fundamental y que ya hemos comentado más arriba (el tener que asumir el papel de una figura con autoridad), pero la segunda es, precisamente, la temporalidad. Desde su óptica, en el contexto de WST, puede ser muy importante que uno de los dos elementos del condicional ocurra antes que el otro.

Con el propósito de comprobar este último supuesto, Alkhalifa presenta una tarea semejante a las versiones analizadas en Cheng y Holyoak (1985). Así, trabaja con dos grupos de participantes. Todos ellos, independientemente del grupo al que pertenezcan, ejecutan una versión abstracta y una versión temporal (entre el antecedente y el consecuente de la regla hay una distancia en el tiempo) de WST. La diferencia entre la labor a realizar por ambos grupos reside en que uno de ellos se enfrenta a la versión abstracta primero y el otro, por el contrario, realiza la versión temporal antes que la abstracta. En la versión temporal, los participantes tienen que imaginar que se encuentran frente a un tablero con dos botones, uno rojo y otro azul. Cuando se presiona un botón, éste se ilumina y evidencia — durante cinco minutosque ha sido pulsado durante cinco minutos, pues se apaga después de ese tiempo. No obstante, justo cuando se apaga, aparece en el tablero una fotografía de un pájaro o de una flor. Con este contexto en mente, el participante tiene que pensar en una sala de clases en la que se pueden observar estas cuatro situaciones 
A.- El estudiante A tiene iluminado el botón rojo.
B.- El estudiante B tiene iluminado el botón azul.
C.- El estudiante C tiene un pájaro en el tablero.
D.- El estudiante D tiene una flor en el tablero.

La regla es ésta

Si se presiona el botón rojo, entonces, la flor tiene que aparecer cinco minutos después de esa acción.

El participante debe tener en cuenta que a los estudiantes no les gusta ser interrumpidos cuando están trabajando. Sin embargo, tiene que comprobar la regla y, para ello, necesita decidir si va a preguntarle a algún estudiante qué botón ha presionado, si simplemente va a observar las fotografías que van a aparecer cuando se apaguen los botones encendidos o si va a combinar estas dos posibilidades.

Según sus resultados, cuando la versión temporal se ejecuta después de la abstracta, el número de sujetos que seleccionan las tarjetas correctas desciende significativamente. Empero, cuando se invierte el orden y la versión temporal se realiza primero, la ejecución mejora, también significativamente. Alkhalifa interpreta, a partir de estos datos, que, aunque sus supuestos iniciales se centraban en la temporalidad, el elemento más importante a considerar en WST es la direccionalidad y no el tiempo. ${ }^{5}$ Pero, a nuestro juicio, la temporalidad es esencial para comprender las relaciones condicionales. Evidentemente, que un participante ejecute una versión con anterioridad a otra puede influir en la dirección de su pensamiento, pero ello no implica que la temporalidad sea irrelevante. Obviamente, las representaciones mentales que un participante se construye cuando interpreta las instrucciones de una de las dos versiones tienen que condicionar las representaciones mentales que ese mismo sujeto elabora cuando se enfrenta, en segundo lugar, a la otra versión, y esto es válido para los dos grupos experimentales de Alkhalifa, sea cual sea la versión por la que empiecen. Desde nuestro punto de vista, es indiscutible que las versiones de WST con reglas que establecen una dimensión temporal entre el antecedente y el consecuente suelen realizarse correctamente con mayor frecuencia. Vamos a comprobarlo a continuación, en el apartado siguiente, por medio del análisis de algunas reglas características de versiones muy conocidas de WST. Después, nos

\footnotetext{
${ }^{5}$ Ello lo demuestra, en su opinión, el hecho de que la versión temporal obtenga peores resultados cuando se ejecuta en segundo lugar. Como se puede deducir de su explicación, si el factor determinante fuera el tiempo, no debería descender el número de respuestas lógicamente válidas al realizarse la versión temporal después de la abstracta, pues debería ser indiferente el orden, y la dimensión temporal tendría que facilitar la comprensión de la labor a realizar tanto si los participantes se enfrentan a la versión temporal antes como si lo hacen a continuación. No obstante, no podemos olvidar que los resultados nos muestran que, cuando el participante realiza la versión temporal primero, mejoran sensiblemente los resultados de la versión abstracta, pudiéndose aducir, además, que la versión temporal, realizada con anterioridad a la abstracta, parece arrojar mejores resultados que los habituales en la literatura para esta última. Estos hechos, sin duda, necesitan una explicación por sí mismos.
} 
ocuparemos de aquellas versiones que no presentan dimensión temporal alguna en su regla condicional y que, a pesar de ello, también ofrecen elevados índices de respuesta válida desde el punto de vista lógico, debido a que se establece en ellas una relación de inclusión. Nuestro objetivo es demostrar que la temporalidad y la relación de inclusión son las dos características que pueden mejorar la ejecución de una versión determinada de WST. Comenzamos, pues, con nuestros argumentos sobre la temporalidad.

\section{APOYOS EXPERIMENTALES PARA LA TESIS DE LA TEMPORALIDAD}

La base de nuestros supuestos radica en la idea de que la distancia cronológica entre el antecedente y el consecuente ayuda a entender adecuadamente las afirmaciones condicionales, opinión que podemos fundamentar en los resultados de una gran cantidad de trabajos sobre WST que han sido publicados en el área de la ciencia cognitiva. Veamos algunos de ellos.

Comenzamos por uno que, aparentemente, parece contradecir nuestro planteamiento. Wason y Shapiro (1971) propusieron un escenario en el que se hablaba de cuatro días de la semana y de cuatro viajes. Sus tarjetas mostraban estas posibilidades: "Manchester", "Leeds", "Tren" y "Automóvil", siendo la regla similar a ésta

\section{Cada vez que voy a Manchester, viajo en automóvil.}

Como se puede apreciar, no es posible notar ninguna dimensión temporal en esta regla, ya que viajar a Manchester y hacerlo en automóvil son dos actos que se realizan al unísono. No obstante, contra nuestra tesis, diez de los dieciséis sujetos experimentales que se enfrentaron a esta versión de WST eligieron las tarjetas correctas ("Manchester" y "Tren"). A pesar de ello, consideramos que este hecho no supone ningún obstáculo ni ninguna dificultad severa para nuestros supuestos, fundamentalmente, por dos motivos:

1.- No podemos aceptar que diez sujetos - frente a dieciséis - signifiquen un resultado indiscutible y definitivo.

2.- Manktelow y Evans (1979) replicaron este experimento de Wason y Shapiro

(1971) de modo exacto y no obtuvieron resultados positivos (en nuestra opinión, porque la temporalidad no está presente en la regla).

Sin embargo, en un experimento de Asensio, Martín Cordero, García Madruga y Recio (1990), los porcentajes son bastante más nítidos. La idea de ellos era mostrar que el contenido temático mejora de manera evidente la ejecución de WST y, en su trabajo, encontramos una versión que apoya totalmente nuestro planteamiento. En tal versión, los sujetos experimentales eran adolescentes y las tarjetas mostraban individuos con diferentes edades (mayores y menores de edad) por una cara, y medios de transporte (automóviles y bicicletas), por la otra. La regla se encontraba relacionada con la edad requerida para poder conducir automóviles y era parecida a la siguiente

Si conduces un automóvil, entonces has de ser mayor de edad

Un número considerable de participantes eligió las tarjetas válidas desde el punto de vista lógico en este caso. Para nosotros, ello sucedió porque, temporalmente, es necesario ser mayor de edad antes de estar habilitado para conducir. Así, esta 
perspectiva diacrónica permite entender que lo que la regla establece es verdaderamente una relación condicional.

Los problemas de Gigerenzer y Hug (1992), utilizados también por Almor y Sloman (2000), establecen igualmente una distancia temporal evidente. Dichos problemas nos revelan que las selecciones de los participantes, habitualmente, son excelentes para reglas de este tipo

Si un empleado disfruta de un día libre de lunes a viernes, entonces dicho empleado tiene que haber trabajado el fin de semana.

Es indudable que, para conseguir un día libre, es indispensable haber trabajado durante el fin de semana (antes). Podemos decir, por consiguiente, que esta versión continúa apoyando nuestra idea referente a la necesidad de una diferencia cronológica entre el antecedente y el consecuente de la regla para mejorar los porcentajes de respuesta correcta en WST. Esta diferencia cronológica es esencial para el participante, pues, sin ella, es más difícil interpretar la regla como desea el investigador que lo haga.

Un esquema diacrónico similar se presenta también en el experimento $\mathrm{N}^{\circ} 2,{ }^{6}$ de Fiddick, Cosmides y Tooby (2000) que describe un contexto en el que algunos antropólogos visitan la tribu Kalama y obsequian sus miembros con chaquetas luminosas anaranjadas. Aquí la regla es

Si marchas de cacería, entonces tienes que vestirte con estas chaquetas para que no te disparen

Obviamente, en esta versión, el individuo tiene que vestirse con la chaqueta con anterioridad a salir de caza. El experimento 3 de Fiddick, Cosmides y Tooby mantiene la misma estructura: hay también una tribu indígena en su contexto, pero, ahora, el antropólogo regala guantes de caucho. Él sabe que en la tribu se fabrican dardos venenosos y que, en el proceso de elaboración, el veneno puede entrar en contacto con la piel. La afirmación en este experimento es

Si elaboras dardos con veneno, entonces es necesario que utilices guantes de caucho.

Es también manifiesta la relación temporal en esta regla. En ella se indica que el sujeto tiene que enfundarse las manos con guantes de caucho con carácter previo a manipular los dardos.

Cosmides (1989) nos habla de una tribu polinesia, la Kaluame, que posee leyes muy estrictas. Una de esas leyes hace referencia a la raíz de cassava, un afrodisíaco realmente potente y que transforma en irresistible para las mujeres al hombre que la consume. El problema es que la raíz de cassava no es precisamente abundante y, para

\footnotetext{
${ }^{6}$ Podemos presentar una explicación parecida para el experimento 1 de Fiddick, Cosmides y Tooby (2000). En este experimento, un granjero sudamericano, cuando termina de cosechar, nota que le han sobrado papas y viaja a un pueblo vecino con su excedente. En el pueblo, habla con cuatro sujetos (representados mediante las cuatro tarjetas de esta versión de WST), los cuales le dicen: Si me das papas, entonces te daré maíz. Así, para obtener maíz, el granjero tiene que previamente entregar papas.
} 
poder seguir contando con ella como recurso y que no se agote, los solteros tienen totalmente prohibido comerla. Puesto que los hombres de la tribu Kaluame tatúan sus rostros cuando se casan, los ancianos de la tribu decretaron una norma parecida a la que figura a continuación

Si un varón se alimenta con raíz de cassava, entonces debe tener tatuada su cara.

Es evidente, por tanto, que - en esta historia - una acción previa que debe realizar un hombre para poder comer raíz de cassava es contraer matrimonio y, de este modo, tatuarse el rostro.

Manktelow y Over (1991) usaron una regla bastante semejante a la del experimento 3 de Fiddick, Cosmides y Tooby. La regla era similar a ésta

Si tú limpias sangre derramada, entonces tienes que ponerte guantes de goma.

De nuevo, podemos observar en esta regla una dimensión temporal. Sólo se puede limpiar la sangre si, con anterioridad, las manos han sido protegidas con guantes de goma. ${ }^{7}$

Sin embargo, aún nos queda un problema por resolver: existen ciertas versiones de WST en las que también se obtienen resultados óptimos y en las que, a pesar de ello, no es posible detectar ninguna forma de temporalidad en ellas. En el apartado siguiente, nos ocupamos de este asunto.

\section{LAS RELACIONES DE INCLUSIÓN EN CUANTO FACTORES QUE FACILITAN LA EJECUCIÓN DE WST EN VERSIONES SINCRÓNICAS}

A partir de las explicaciones precedentes, podemos decir que el participante en las versiones abstractas iniciales de WST no comprende que el enunciado expresado en la regla es un enunciado condicional. La presencia de la estructura "si..., entonces..." en una afirmación, no garantiza que los sujetos necesariamente comprendan que tal afirmación establece una relación condicional. En nuestra opinión, es más importante otro factor: la existencia de una distancia diacrónica entre sus dos términos. Desde este punto de vista, se desvanece el misterio que

\footnotetext{
${ }^{7}$ Naturalmente, podríamos revisar otras muchas versiones de WST con contenido temático, pues el número de investigaciones sobre esta tarea que han aparecido en las últimas décadas es muy elevado. No obstante, estamos completamente convencidos de que, si lo hiciéramos, no podríamos añadir mucha más información a la ya expuesta, ya que la mayor parte de las versiones en las que los participantes prefieren las tarjetas válidas lógicamente suele presentar dimensiones temporales o perspectivas diacrónicas más o menos similares a las comentadas en este apartado. Si a esto unimos que, por ser tan considerable la cantidad de investigaciones que se han presentado en los últimos tiempos, el análisis de todas ellas aumentaría de modo desproporcionado la extensión de este trabajo, creemos que la opción más oportuna es aceptar las evidencias que acabamos de proponer como lo suficientemente concluyentes - al fin y al cabo, proceden de textos emblemáticos en el área- y como apoyos firmes para nuestra tesis relativa a que la distancia temporal entre el antecedente y el consecuente es la que, básicamente, permite al participante comprender adecuadamente cuál es el verdadero sentido de la regla.
} 
acompaña a las versiones abstractas de WST, pues, en ellas no se puede apreciar ninguna distancia temporal entre el antecedente y el consecuente. En las versiones abstractas, simplemente, se relaciona una letra vocal con un número par y esta conexión es sincrónica. Por esta razón, es difícil para el sujeto notar que está ante un enunciado condicional. Por el contrario, puede pensar, por ejemplo, que se encuentra ante una conjunción, ya que los términos de las conjunciones lógicas muy a menudo se dan al mismo tiempo.

De esta manera, el enunciado "si en una tarjeta hay una vocal en una cara, entonces hay un número par en la otra" puede, como decimos, no ser entendido como una oración condicional. Ahora bien, consideramos que es importante aclarar que no defendemos que, para poder utilizar un condicional con propiedad, sea necesario establecer una dimensión temporal en él. De hecho, es obvio que la regla en las versiones iniciales de WST es un enunciado condicional y que el investigador espera que sea entendido por el sujeto como tal. Lo único que sostenemos es que el individuo se suele equivocar en las versiones abstractas y no en aquellas cuyo contenido temático implica una distancia temporal. En estas últimas, en nuestra opinión, no yerra porque la temporalidad le ayuda a comprender la regla. En ningún caso estamos diciendo que la temporalidad sea una característica intrínseca del condicional ni que éste se utilice de modo ilegítimo cuando el elemento temporal no está presente.

Debemos admitir que existe la posibilidad de que un enunciado condicional no caracterizado por una distancia temporal entre su antecedente y su consecuente sea interpretado correctamente. La temporalidad sólo es un factor que, sencillamente, nos ayuda a construir la representación mental adecuada, pero, a pesar de ser el que con más frecuencia lo hace, no es el único factor que puede desempeñar esa función. En este sentido, deben recordarse los planteamientos de Yachanin y Tweney (1982), de Margolis (1987) y de Nickerson (1996) ya comentados. No obstante, la inquietud mayor puede provocarla, a nuestro juicio, la investigación de Van Duyne (1974) quien comparó, mediante dos versiones (una abstracta y otra temática) cuatro esquemas lógicamente equivalentes. Las reglas temáticas de su experimento eran semejantes a éstas

1.- Condicional: Si eres un estudiante de Filosofía, entonces estás en Cambridge.

2.- Universal: Todos los estudiantes de Física están en Oxford.

3.- Disyuntiva: O no estudias Francés o estás en Londres.

4.- Conjuntiva: No puede darse el caso de que estudies Psicología y no estés en Glasgow.

Estos cuatro enunciados mantienen entre sí una relación de equivalencia lógica, y ello puede notarse fácilmente si una misma oración la expresamos según estas cuatro

\footnotetext{
${ }^{8}$ Como hemos apuntado más arriba, en López Astorga (2008b) ya ofrecimos argumentos a favor de la posible interpretación de la regla como conjunción en las versiones abstractas iniciales. Véase tal trabajo para una explicación más detallada al respecto.
} 
estructuras. A título exclusivamente ilustrativo, podemos pensar, por tomar un ejemplo cualquiera, en el célebre enunciado de la paradoja de los cuervos negros de Hempel (1945). Tal enunciado, manteniendo un significado idéntico, podría adoptar estas cuatro formas

A.- Condicional: Si esto es un cuervo, entonces es negro.

B.- Universal: Todos los cuervos son negros.

C.- Disyuntiva: $O$ esto no es un cuervo o es negro.

D.- Conjuntiva: No puede darse el caso de que esto sea un cuervo y no sea negro.

Como se puede apreciar, A, B, C y D son no sólo equivalentes lógicamente sino también semánticamente idénticos. Sólo cambia su formato, pues, en realidad, transmiten el mismo mensaje. Sin embargo, lo relevante para nosotros aquí es que, a pesar de que en el experimento de Van Duyne (1974) sobre las carreras universitarias y las ciudades no se da ningún tipo de temporalidad, ya que un sujeto estudia una carrera y lo hace en una ciudad al mismo tiempo, se halló en este experimento claras diferencias de ejecución entre las versiones abstractas y temáticas de las condiciones condicional y universal. En estas dos últimas condiciones se obtuvieron mejores resultados cuando los participantes trabajaron con contenido temático. ${ }^{9}$

Empero, lo que reviste interés para nosotros ahora es que: a) El trabajo de Van Duyne (1974) demuestra que no siempre procesamos las expresiones del lenguaje natural según las formas lógicas con las que se presentan (Deaño, 1999) lo cual apoya nuestros supuestos, ya que, en definitiva, una de las ideas fundamentales que venimos sosteniendo en este trabajo es que los principales problemas de algunas versiones de WST residen en que los participantes no entienden el enunciado que aparece en la regla como se esperaría a partir de su forma lógica, y b) También evidencia que es posible comprender correctamente una afirmación condicional en la que no esté presente la temporalidad.

A partir de lo que llevamos expuesto, claro está que b) necesita una explicación. Disponemos de ella, pues pensamos que la lógica nos concede los recursos suficientes para entender lo que sucede en el experimento de Van Duyne. Como sabemos, la lógica no es sólo el cálculo proposicional o la lógica de predicados de primer orden. Dentro del área de la lógica nos encontramos, también, con la teoría de conjuntos, igualmente denominada "lógica de clases". Si atendemos a los recursos que nos proporciona la teoría de conjuntos, podemos suponer que el sujeto experimental, para la primera regla

\footnotetext{
${ }^{9}$ Ya en López Astorga (2004) comentamos el análisis que Stenning y Van Lambalgen (2001) realizaron de los resultados de Van Duyne (1974). Para ellos, consecuencias importantes se derivan del trabajo de Van Duyne, puesto que nos muestran que las formas lógicas de las expresiones no garantizan, por sí mismas, que el sujeto interprete correctamente tales expresiones. Los cuatro enunciados de Van Duyne, que eran, como hemos indicado, equivalentes desde el punto de vista formal, no fueron entendidos en la misma medida por sus participantes. A juicio de Stenning y Van Lambalgen, esto puede deberse, por ejemplo, a fenómenos tales como que, generalmente, los sujetos no captan la correspondencia lógica que puede existir entre un condicional y una disyunción con el primer término negado.
} 
de Van Duyne, pudo construirse una representación mental en una dirección semejante a ésta: el conjunto de los estudiantes de Filosofía es un subconjunto del conjunto de los estudiantes de Cambridge. Sin duda, una representación mental como ésta parece ser más que suficiente para comprender que la relación entre los dos términos de la primera regla es una relación condicional, ya que refleja con claridad la idea que se transmite. Lo mismo podemos decir con respecto a la segunda regla, la universal, pues permite una representación mental similar y que bien puede ser: el conjunto de los estudiantes de Física es un subconjunto del conjunto de los estudiantes de Oxford.

No obstante podríamos preguntarnos, legítimamente, por qué los sujetos experimentales de Van Duyne no fueron capaces de construirse representaciones mentales análogas para las reglas tercera y cuarta. Sin embargo, la respuesta a la pregunta para la regla tercera, puede ser obvia si tenemos en cuenta las reflexiones de Stenning y Van Lambalgen (2001) a las que hemos hecho referencia más arriba, relativas a lo complejo que resulta encontrar equivalencias entre enunciados con disyunciones y condicionales. Procesar disyunciones, además, es una actividad que para el individuo requiere, abusando de la terminología introducida por Sperber y Wilson (1986), un mayor esfuerzo cognitivo que procesar condicionales o enunciados universales de índole similar a la de los ejemplos con contenido temático que trabaja Van Duyne. Con respecto a la conjunción lógica que caracteriza la estructura formal de su cuarto enunciado, creemos que no son necesarios análisis profundos para tomar conciencia de que también se trata de una afirmación mucho más compleja que las dos primeras y para la que es bastante más difícil elaborarse la representación mental que verdaderamente corresponde a lo que ella expresa literalmente.

En realidad, el hecho de que los participantes de Van Duyne no logren buenos resultados con respecto a sus enunciados tercero y cuarto no debe preocuparnos demasiado, pues nos revela, una vez más, que los sujetos no siempre procesan las informaciones lingüísticas del modo que sería apropiado para una formalización lógica correcta, lo cual es completamente consistente con nuestras tesis. Lo interesante de su experimento es, como se deriva de lo expuesto, que una versión de WST en la que se manifieste con claridad una relación de inclusión entre los dos términos de la regla debe ser resuelta adecuadamente por sus participantes. Esto lo podemos deducir, sin mayores esfuerzos teóricos, a partir de su primera regla, que es, sin duda, la fundamental para nuestro estudio.

Pero podría pensarse que esta primera regla de Van Duyne nos revela el aspecto que es verdaderamente trascendental para una interpretación correcta de los enunciados condicionales, esto es, que la existencia de una relación de inclusión entre sus términos favorece en mayor medida que la temporalidad, una comprensión adecuada de los mismos. De hecho, podría defenderse que la temporalidad sólo es la causa de un procesamiento apropiado de los condicionales que la presentan aparentemente. No obstante, trabajos como el ya mencionado de Alkhalifa arrojan pocas dudas al respecto. Se trata de un experimento que en su condición temporal 
presenta mejores resultados que en su condición abstracta y en el que la condición temporal no propone una regla en la que se pueda fácilmente detectar una relación de inclusión, ya que no resulta en absoluto sencillo - más bien, parece totalmente imposible - elaborar una representación mental en la que los botones rojos sean los elementos de un hipotético subconjunto que pertenezca al conjunto de las fotografías de flores que aparecen en un tablero. Difícil es también, en este sentido, por indicar otro ejemplo, entender como relación de inclusión el vínculo que puede existir entre las papas y el maíz en el, también nombrado más arriba, experimento 1 de Fiddick, Cosmides y Tooby.

Es, por tanto, evidente, que la dimensión temporal desempeña una importante función en el procesamiento de los condicionales. Ahora bien, lo que sí tenemos que admitir es que existen versiones de WST en las que se dan los dos elementos, la temporalidad y la relación de inclusión. Tal es lo que sucede, por pensar en una versión que también hemos comentado, en el experimento relativo a la tribu Kaluame de Cosmides. Su regla condicional, además de plantear, como dijimos en el apartado anterior, una distancia temporal entre el antecedente y el consecuente, puede conducir a una representación semejante a ésta: el conjunto de los varones que se alimentan con raíz de cassava es un subconjunto del conjunto de los varones con un tatuaje facial. Ante estos casos, en los que la versión concreta cuenta con las dos propiedades necesarias para interpretar adecuadamente el condicional, sólo podemos decir que el participante va a mostrar una ejecución excelente con un grado bastante alto de probabilidad.

En cualquier caso, nuestra explicación puede servirnos, incluso, para explicar lo sucedido con el experimento de Wason y Shapiro (1971) sobre las ciudades y los medios de transporte y con su réplica en el trabajo de Manktelow y Evans (1979). Diez de los dieciséis sujetos experimentales de Wason y Shapiro eligieron las tarjetas correctas porque, si bien en su versión de WST no se puede apreciar ninguna dimensión temporal, es posible construirse para ella una representación mental basada en una relación de inclusión y pensar que, en el contexto que describe, el conjunto de los viajes a Manchester puede ser un subconjunto del conjunto de los viajes en automóvil. Es obvio que hacerlo requiere un mayor costo cognitivo que el que es necesario para hacer lo mismo con respecto a la primera afirmación de Van Duyne. Ciertamente, podemos imaginarnos conjuntos de estudiantes sin dificultad y, aunque también podemos imaginarnos conjuntos de viajes, esto último es bastante más complicado, pues la idea de conjunto de viajes bien puede ser contraintuitiva. No se precisa, por tanto, el mismo grado de abstracción en las dos versiones, por lo que esta diferencia puede explicar los peores resultados de la réplica de Manktelow y Evans.

No podríamos presentar, empero, argumentos análogos para las versiones iniciales abstractas de WST, a las que resulta mucho más difícil aplicarles la lógica de clases. El conjunto de las letras vocales y el conjunto de los números pares son, de acuerdo con el sentido común, conjuntos disjuntos, es decir, conjuntos sin ningún elemento en común, y 
entre conjuntos disjuntos es imposible hablar de una relación de inclusión. Esto nos ayuda a comprender, en mayor medida aún, por qué las versiones abstractas iniciales de WST no suelen resolverse adecuadamente, ya que, en ellas, no sólo no se da la temporalidad, sino que tampoco se pueden apreciar relaciones de inclusión.

Nuestras tesis se ven apoyadas, además, por lo que sucede con otras versiones de WST que han mostrado resultados negativos o resultados positivos muy discretos y no muy claros. En Manketelow y Evans se propone un enunciado parecido a éste

\section{Si como bacalao, entonces bebo ginebra.}

Como se puede apreciar, en esta regla no existe distancia temporal clara entre el antecedente y el consecuente, pues, no se dice que se beba ginebra después de comer bacalao, sino que se bebe ginebra siempre que se come bacalao, sin precisar en absoluto en qué momento se hace. Además, no es posible entender la relación entre el bacalao y la ginebra en términos de la lógica de clases, pues no podemos suponer que uno de los términos es un subconjunto del otro. Así, no resulta extraño que versiones como éstas vayan acompañadas de porcentajes muy bajos de selección de tarjetas válidas desde el punto de vista lógico. ${ }^{10}$

Otra regla controvertida es la que propusimos en nuestro experimento 2 (2004). La regla era ${ }^{11}$

Si te matriculas en la Facultad de Filosofía, entonces tienes que presentar una fotocopia compulsada de tu título de Bachillerato.

Con esta versión de WST con contenido temático obtuvimos como resultado que la selección correcta fue la más elegida, pero, para nosotros, no estuvimos ante un porcentaje óptimo de selección, ya que, en realidad, sólo fueron seis, de entre veintidós, los alumnos que se inclinaron por las tarjetas válidas desde el punto de vista lógico (con lo que tuvimos dieciséis que erraron en su respuesta). Esta situación también es explicable, no obstante, desde nuestros supuestos. Por una parte, la opción correcta lógicamente es la preferida porque tanto una perspectiva temporal como una relación de inclusión pueden observarse en esta regla. Hay que cursar el Bachillerato

\footnotetext{
${ }^{10}$ Creemos importante insistir, en cualquier caso, en que no estamos defendiendo que la temporalidad y la relación de inclusión sean los únicos elementos que pueden ayudar al sujeto a interpretar adecuadamente una versión de WST. A pesar de que, para nosotros, son las características fundamentales, tenemos que aceptar que, evidentemente, los factores propuestos por Yachanin y Tweney (1982), por Margolis (1987) y por Nickerson (1996) — a los que ya hemos hecho mención en más de una ocasión - también pueden influir en la construcción de representaciones mentales que realiza el participante. En este sentido, cabe, además, admitir que el hecho de que comer bacalao bebiendo ginebra sea una situación no muy corriente y casi inverosímil también debe manifestarse en los bajos porcentajes de esta versión. Con respecto a esta última afirmación. Cfr., Wason y Green (1984) y López Astorga (2004).

${ }^{11}$ Este experimento fue aplicado a alumnos de Educación Media en España, país en el que el término "Bachillerato" hace referencia a los estudios secundarios que un estudiante debe cursar antes de poder acceder a la Universidad, y "compulsada", significa algo similar al concepto de "legalizada", utilizado en otros países hispanohablantes.
} 
en España antes de poder ingresar a estudios universitarios (dimensión temporal) y el conjunto de estudiantes matriculados bien puede ser un subconjunto del conjunto de los estudiantes que presentan fotocopia compulsada de su título de Bachillerato. Sin embargo, por otra parte, aunque la opción correcta es la respuesta mayoritaria, no es elegida por un porcentaje demasiado elevado de participantes porque, en realidad, la regla establece que matricularse y presentar la fotocopia del título de Bachillerato son dos acciones que deben realizarse al unísono (se desvanece la temporalidad) y en una Facultad de Filosofía en España, generalmente, sólo están matriculados los estudiantes que han presentado la fotocopia compulsada del título de Bachillerato (lo que supone que es muy difícil entender esos dos conjuntos - el de los alumnos matriculados y el de los alumnos que presentan la fotocopia compulsada de su título de Bachillerato- uno como un subconjunto del otro, pues son, en la práctica, conjuntos idénticos con exactamente los mismos elementos).

Así, tenemos que, si bien no es imposible vislumbrar una dimensión diacrónica y una relación de inclusión en esta versión de WST, es mucho más complejo que en otras. Esto explica que el par de tarjetas correcto, a pesar de ser la opción más elegida, sólo sea seleccionado por un número no muy relevante de participantes.

Parecen, por consiguiente, confirmarse nuestras tesis iniciales. Las versiones de WST con contenido temático concreto que se ejecutan adecuadamente desde el punto de vista lógico son, fundamentalmente, las que presentan una relación diacrónica entre el antecedente y el consecuente de su regla (así nos lo demuestra la mayor parte de las versiones que han sido ejecutadas con éxito) o aquéllas en las que es posible establecer una relación de inclusión entre sus términos (pues tal relación es lo único que, a nuestro juicio, puede explicar resultados como los obtenidos por Van Duyne). El tener un contexto enriquecido, el solicitar asumir el rol de una autoridad, el tener que buscar infractores, son, sin duda, también factores que pueden incidir en una mejora de los porcentajes de selección válida, pero, como hemos podido comprobar en nuestra exposición, la temporalidad y las relaciones de inclusión parecen ser los elementos más determinantes.

CONCLUSIONES: HACIA UNA REFLEXIÓN CRÍTICA EN EL ÁMBITO DE LA CIENCIA COGNITIVA

Sabemos que las leyes de la naturaleza física pueden ser universales y necesarias, ${ }^{12}$ pero es evidente que las leyes que rigen los fenómenos humanos —si es que tales leyes existen- difícilmente pueden tener tales características. Quizás, el problema fundamental de WST sea que, en muy raras ocasiones, todos los participantes que se enfrentan a una de sus versiones, sea ésta con contenido temático o abstracta, seleccionan las mismas tarjetas. Por tanto, parece que no es posible extraer conclusiones

\footnotetext{
${ }^{12} \mathrm{Al}$ menos en la perspectiva de la mecánica clásica, pues somos conscientes de que quizás, en el presente, una afirmación de este tipo deba ser matizada y tomar en consideración, como mínimo, las posibles inconsistencias que pueden detectarse a nivel microfísico. (Véase, con respecto a este último aspecto, por ejemplo, Tahko, 2009).
} 
definitivas o, por lo menos, absolutas, tras una revisión de los resultados de cualquier versión de WST, acerca del comportamiento intelectual de las personas o de la manera en que realizan su razonamiento los seres humanos, ya que tales conclusiones deben ser siempre, a nuestro juicio, provisionales. Y es que debemos proceder con cautela cuando estudiamos la cognición humana. ${ }^{13}$

Sin embargo, consideramos que hay un aspecto trascendental en este debate que no debe ser obviado. Defendemos aquí, manteniendo consistencia y coherencia con otros trabajos representativos (Cohen, 1981; Almor y Sloman, 2000; Stenning y Van Lambalgen, 2002) y con otros anteriores estudios nuestros, que las dificultades de los sujetos que se enfrentan a WST son dificultades de interpretación, no de razonamiento como se podría pensar en un primer momento. Los sujetos que resuelven mal WST no lo hacen porque realizan inferencias erradas, sino, simplemente, porque no comprenden la labor que se les está proponiendo. Los problemas en WST no son, por tanto, de razonamiento lógico, sino de procesamiento de la información. Esto es muy importante no olvidarlo para no caer en confusiones y no sucumbir a la tentación de pensar que la lógica formal no es realmente formal, puesto que - como podría pensarse en virtud de los buenos resultados que se obtienen con algunos contenidos temáticos - cuando se narra una historia concreta, se crea un escenario que nos permite extraer inferencias más acertadas.

Éste es un error de apreciación que puede soslayarse fácilmente si tenemos en cuenta que la complejidad de WST se encuentra en la elaboración de representaciones mentales a partir de sus instrucciones y no en el razonamiento lógico ulterior que tiene lugar cuando tales representaciones ya han sido elaboradas. Desde este punto de vista, la lógica formal permanece intacta y no alterada por el contenido, pues éste sólo parece influir en la interpretación de la información linguiística que se le transmite a un sujeto. Las estructuras descritas en las reglas y principios lógicos elementales no son lo que se puede cuestionar a partir de los resultados de WST. ${ }^{14}$

\footnotetext{
${ }^{13}$ Asentadas estas bases, podemos afirmar que, a pesar de ello, es posible observar en el pensamiento humano ciertas tendencias o regularidades y que WST puede revelarnos algunas. Lo esencial que nos muestra WST es que las personas, por elevado que sea su nivel cultural o educativo, no necesariamente interpretan la información que se les transmite del modo literal en que, en principio, deberían hacerlo (recordemos, en este sentido, que diversas versiones de WST han sido presentadas a individuos con formación universitaria y que éstos han tendido a incurrir en los mismos errores que habitualmente se cometen cuando se realiza esta tarea). En concreto, nos evidencia que los enunciados condicionales son de muy difícil comprensión para la mayoría de los sujetos si no van acompañados de un contexto adecuado.

${ }^{14}$ De lo dicho no debe deducirse, empero, que defendemos que el pensamiento humano se atiene exclusiva y rigurosamente a las reglas y principios fundamentales de la lógica clásica, sea ésta proposicional o de predicados de cualquier orden. Nuestros argumentos sólo hacen referencia a la idea de que, si en la mente humana suceden ciertos eventos ilógicos que guían y orientan el pensamiento ignorando las prescripciones lógico-formales, WST no es la tarea más idónea para probarlo. A menudo, los investigadores sólo se han centrado en las respuestas que ofrecen los participantes, comparándolas únicamente con la regla de la versión concreta ejecutada y presentando, de este modo, mediante análisis tan limitados, conclusiones acerca de las capacidades lógico-racionales de dichos participantes. Desde
} 
Parece, en definitiva, que el ámbito básico en el que deben centrarse en el momento presente los teóricos de las ciencias cognitivas es el relativo al procesamiento de la lengua natural. Mucho conocemos acerca de la lógica, de los sistemas axiomáticos, de los cálculos lógicos y, por supuesto, también sobre las propuestas de lógicas no clásicas, como la lógica modal o la lógica deóntica. No obstante, ninguna tarea de razonamiento nos va a servir para verificar cuáles de estos sistemas describe mejor el funcionamiento de la mente humana, si es que alguno de ellos lo hace verdaderamente, hasta que no estemos totalmente seguros de que los participantes en esa tarea comprenden sus instrucciones perfectamente. Por eso, necesitamos, acuciantemente, focalizar nuestra atención en los procesos por medio de los cuales construimos nuestras representaciones mentales.

$$
\begin{array}{r}
\text { Universidad de Los Lagos* } \\
\text { Departamento de Educación } \\
\text { Avda. Alcalde Fuchslocher 1305. Osorno (Chile) } \\
\text { m.lopez@ulagos.cl }
\end{array}
$$

\section{BIBLIOGRAFÍA}

Alkhalifa, E. M. 'Egoistic reasoning 'in time' in the selection task", in Proceedings of the Third International Conference on Cognitive Science, ICCS2001. Beijing, (2001):109-113. http://www.silvertair.com/papers/Egoistic\%20Formatted.doc.

Consultado: Septiembre 6 del 2010.

Almor, A.; Sloman, S. A. "Reasoning versus text processing in the Wason selection task -a non - deontic perspective on perspective effects", in Memory \& Cognition 28 (2000):1060-1069.

Asensio, M.; Martín Cordero, J. I.; García Madruga, J.; Recio, J. "Ningún iroqués era mohicano: la influencia del contenido en las tareas de razonamiento lógico", en Estudios de Psicología 43-44 (1990):35-60.

Beller, S. "Deontic reasoning reviewed: psychological questions, empirical findings, and current theories", in Cognitive Processing 11 (2010):123-132.

Cheng, P. W.; Holyoak, K. J. "Pragmatic reasoning schemas", in Cognitive Psychology 17 (1985):391-416.

Cheng, P. W.; Holyoak, K. J.; Nisbett, R. E.; Oliver, L. M. "Pragmatic versus syntactic approaches to training deductive reasoning", in Cognitive Psychology 18 (1986) :293-328.

Cohen, L. J. “Can human irrationality be experimentally demonstrated?", in Behavioral and Brain Sciences 4 (1981):317-370.

nuestra óptica, proceder así exclusivamente es un error manifiesto, pues significa obviar todos los procesos mentales, no necesariamente inferenciales, que tienen lugar antes de que un sujeto seleccione una tarjeta. En concreto, para nosotros, tal actitud es una manera de proceder que ignora un proceso tan importante en la actividad intelectual como lo es el de la comprensión del discurso, comprensión que debe realizarse adecuadamente para después realizar las inferencias lógicas previstas por el experimentador. Si tal comprensión no se realiza correctamente, el sujeto puede continuar razonando siguiendo estrictamente los principios lógicos y, sin embargo, llegar a conclusiones erróneas por partir de premisas que no se corresponden literalmente con las instrucciones transmitidas. 
Cosmides, L. "The logic of social exchange: has natural selection shaped how humans reason? Studies with the Wason selection task", in Cognition 31 (1989):187-276.

Deaño, A. Introducción a la lógica formal. Madrid: Alianza, 1999.

Evans, J. St. B. T. "Dual-processing accounts of reasoning, judgment, and social cognition", in Annual Review of Psychology 59 (2008):255-278.

Fiddick, L.; Cosmides, L.; Tooby, J. "No interpretation without representation: the role of domain-specific representations and inferences in the Wason selection task", in Cognition 75 (2000):1-79.

Gigerenzer, G.; Hug, K. "Domain-specific reasoning: social contracts, cheating, and perspective change", in Cognition 43 (1992):127-171.

Hempel, C. G. "Studies in the logic of confirmation (I)", in Mind 54 (1945):1-26.

Johnson-Laird, P. N.; Legrenzi, P.; Legrenzi, M. S. "Reasoning and sense of reality", en British Journal of Psychology 63 (1972):395-400.

López Astorga, M. “Los contratos sociales: ¿Un nuevo formalismo ético?”, en Alpha. Revista de Artes, Letras y Filosofía N ${ }^{\circ}$ 30. Osorno: Universidad de Los Lagos (2010): 9-26.

"Tarea de selección: una explicación desde la lógica formal”, en A Parte Rei. Revista de Filosofía 59 (2008b). http://serbal.pntic.mec.es/ cmunoz11/astorga59.pdf Consultado: Septiembre 6 del 2010.

------ "Las cuatro tarjetas y el razonamiento humano", en Ciencia Cognitiva 2:3 (2008a):78-80.

------ Revisión del razonamiento condicional a partir de la tarea de selección. Revising conditional reasoning by means of selection task (Spanish text). Ann Arbor (Michigan): Proquest, Information and Learning Company, 2004.

Manktelow, K. I.; Evans, J. St. B. T. "Facilitation of reasoning by realism: effect or not effect?", in British Journal of Psychology 71 (1979):227-231.

Manktelow, K. I.; Over, D. E. "Social roles and utilities in reasoning with deontic conditionals", in Cognition 39 (1991):85-105.

Margolis, H. Patterns, thinking, and cognition: a theory of judgement. Chicago: The University of Chicago Press, 1987.

"Wason's selection task with a reduced array", in Psycoloquy 11:005. Consultado: Septiembre 6 del 2010. http://www.cogsci.ecs.soton.ac.uk/cgi/psyc/newpsy?11.005

Nickerson, R. S. “Hempel's paradox and Wason's selection task: logical and psychological puzzles of confirmation", in Thinking and Reasoning 2:1. (1996):1-31.

Rutherford, M. D. "Cheater detection is preserved in autism spectrum disorders", in Journal of Social, Evolutionary, and Cultural Psychology. 3:2 (2009):105-117.

Santamaría, C. Introducción al razonamiento humano. Madrid: Alianza, 1995.

Sperber, D.; Wilson, D. Relevance: communication and cognition. Oxford: Blackwell.

Stenning, K.; Van Lambalgen, M. "The natural history of hypotheses about the selection task: towards a philosophy of science for investigating human reasoning". K. Manktelow y M. Chung (Eds.). Psychology of reasoning: historical and theoretical perspectives. Londres: Psychology Press, 2002.

"Semantics as a foundation for psychology: a case study of Wason's selection task", in Journal of Logic, Language and Information 10 (2001):273-317.

Tahko, T. E. "The law of non-contradiction as a metaphysical principle", in Australasian Journal of Logic 7 (2009):32-47.

Van Duyne, P. C. "Realism and linguistic complexity in reasoning", in British Journal of Psychology 65 (1974):59-67. 
Wason, P. C. "Reasoning about a rule", in Quarterly Journal of Experimental Psychology 20. (1968):273-281.

----- "Reasoning". B. Foss (Comp.). New horizons in psychology. Harmondsworth (Middlesex): Penguin, 1966.

Wason, P. C.; Green, D. W. "Reasoning and mental representation”, in Quarterly Journal of Experimental Psychology 36:A (1984):597-610.

Wason, P. C.; Shapiro, D. "Natural and cortived experience in a reasoning problem", in Quarterly Journal of Experimental Psychology 23 (1971):63-71.

Yachanin, S. A.; Tweney, R. D. "The effect of thematic content on cognitive strategies in the four-card selection task", in Bulletin of the Psychonomic Society 19 (1982):87-90. 